\title{
Computer-assisted counting of retinal cells by automatic segmentation after TV denoising
}

\author{
Kristian Bredies ${ }^{1}$, Marcus Wagner ${ }^{2}$, Christian Schubert ${ }^{3}$ and Peter Ahnelt ${ }^{3 *}$
}

\begin{abstract}
Background: Quantitative evaluation of mosaics of photoreceptors and neurons is essential in studies on development, aging and degeneration of the retina. Manual counting of samples is a time consuming procedure while attempts to automatization are subject to various restrictions from biological and preparation variability leading to both over- and underestimation of cell numbers. Here we present an adaptive algorithm to overcome many of these problems.

Digital micrographs were obtained from cone photoreceptor mosaics visualized by anti-opsin immuno-cytochemistry in retinal wholemounts from a variety of mammalian species including primates. Segmentation of photoreceptors (from background, debris, blood vessels, other cell types) was performed by a procedure based on Rudin-OsherFatemi total variation (TV) denoising. Once 3 parameters are manually adjusted based on a sample, similarly structured images can be batch processed. The module is implemented in MATLAB and fully documented online.

Results: The object recognition procedure was tested on samples with a typical range of signal and background variations. We obtained results with error ratios of less than $10 \%$ in 16 of 18 samples and a mean error of less than $6 \%$ compared to manual counts.

Conclusions: The presented method provides a traceable module for automated acquisition of retinal cell density data. Remaining errors, including addition of background items, splitting or merging of objects might be further reduced by introduction of additional parameters. The module may be integrated into extended environments with features such as 3D-acquisition and recognition.
\end{abstract}

Keywords: Mammalian photoreceptor cells, Automatical counting, Adaptive algorithm, Continuous optimization, Total variation denoising

\section{Background} Introduction

The vertebrate retina contains two or more subtypes of photoreceptors and dozens of interneuron types, thus being organized for effective operation at different light levels and at different bands of the sunlight's spectrum. Regional shifts in densities and proportions of the subtypes of photoreceptors and interneurons in the retina have been studied intensively as they are assumed to reflect both the evolution of species and specific adaptations to their lifestyle [1,2]. Deviations within cell density and mosaic regularity from their normal variability are

\footnotetext{
*Correspondence: peter.ahnelt@meduniwien.ac.at

${ }^{3}$ Center for Physiology and Pharmacology, Medical University Vienna,

Schwarzspanierstraße 17, A-1090 Wien, Austria

Full list of author information is available at the end of the article
}

of specific interest for research involved in developmental control or progressive loss of photoreceptor and other cells due to degenerative diseases and other pathologic processes in the visual system.

Obtaining such data implies the acquisition and analysis of a large number of samples, which is often a time-consuming task requiring persons with appropriate training and experience. Given, moreover, the approximately planar organization of retinal layers, the problem of detection and counting of photoreceptor cells is a promising candidate for at least partial automatization. However, while various papers have developed options for mapping and analysis of retinal cell mosaic data, once they are digitized [3-7], approaches to full automatization of the time-consuming procedure of actual identification and counting have been 
rare (we mention [8-10]). Besides the time and computing power required for acquiring two- and threedimensional representations of the tissue of interest, the heterogeneity of the tissue itself is still a major challenge. Many parameters such as tissue thickness/transparency, preparatory and manipulatory distortions change across the retinas, and the cells of interest themselves change in size, shape and spacing. Consequently, for reliable detection of the targets and their differentiation from other items such as debris, other cells, local damage or blood vessels highly adaptive algorithms are required.

The present approach focuses on addressing these problems for the segmentation and counting of photoreceptors. We propose an automatic detection and counting procedure, which is based on Rudin-Osher-Fatemi total variation (TV) denoising [11] with subsequent segmentation. As the comparison with manually collected data shows, this method is able to detect the targets at comparable reliability. In the view of the authors, the main advantage of the method consists in the complete traceability of all data processing steps and its reproducibility independently from a particular software platform. Thus a possibility for standardization and direct comparisons of automatic counts for samples obtained within different environments is provided.

In the present study, the method has been implemented as a MATLAB module and has been applied to single frames (and montages). The method, however, is not limited to the processing of two-dimensional data and can be equally implemented for the analysis of three- or multidimensional data stacks. It could as well be integrated into more comprehensive motorized acquisition setups for stereological sampling or complete mapping of retinal populations.

\section{Retinal image data}

Retinal samples from the following species have been used: Orangutan (Pongo pygmaeus (L. 1760)), Domestic cat (Felis silvestris catus (L. 1758)), Manul (Felis manul (Pallas 1776) ), Eurasian Lynx (Lynx lynx (L. 1758)), Cheetah (Acinonyx jubatus (Schreber 1775)), Jaguar (Panthera onca (L. 1758)), Long-tailed Pangolin (Manis tetradactyla (L. 1766) ) and Black-rumped Agouti (Dasyprocta prymnolopha (Wagler 1831) ) (see Table 1 and Figure 1).

Most of the eyes were obtained from animals delivered to veterinary pathology from zoos and animal parks; some originate from collaborations for other studies $[12,13]$. Post mortem times were between 0.5 and 12 hours. Eyes were enucleated and immersed in $0.01 \mathrm{M}$ phosphate buffer saline (PBS, pH 7.4) with $4 \%$ paraformaldehyde. Some were treated after being opened with a cut along the corneal limbus for faster penetration of fixative. Retina wholemounts were prepared in PBS and flattened by radial cuts, in order to preserve the horizontal and vertical meridian. Cone photoreceptors sensitive to medium/long wavelengths (M-/L-cones) were labeled in isolated Pangolin retina using JH492 antibody, in all other retinas $\mathrm{S}$-cones were labeled using JH455 (both antibodies provided by J. Nathans [14]). In peripheral Jaguar retina, in addition to S-cones horizontal cells are (unintentionally) co-labeled by JH455. Retinal vessels in Orangutan were labeled with rabbit anti-mouse collagen IV (AbD Serotec, 2150-1470). After incubation in primary antisera overnight for up to 3 days visualization was done using goat anti-rabbit igg-biotin (Sigma, B7389), ExtrAvidinperoxidase conjugate (Sigma, E2886) and the diaminobenzidine (DAB) reaction. After washing in PBS, retinas were gradually transferred up to $90 \%$ glycerol, mounted with photoreceptor-side up on a glass slide, and cover slipped.

Manual counting of labeled cones was done within sampling frames of $150 \times 150 \mu \mathrm{m}$ or $300 \times 300 \mu \mathrm{m}$ using an online-video overlay system consisting of Canvas 5 (ACD Systems, USA) software on a Macintosh computer connected with a Hamamatsu 2400 analog camera attached to a Nikon Eclipse microscope. This system allows dual live view of the specimen: through the microscope's optics or on the video image overlaid by the sampling frames. Optional change of focus and illumination supports optimized online identification of cells and exclusion of artifacts by position, form, color and other details.

The images ( 8 or 10 bit grey scale) used for computerassisted cell counting were obtained by using a Photometrix Camera model CH250/A connected to a Nikon Eclipse E600 microscope (magnification factors 200× to $600 \times$ ) using QED Imaging Software (QED Imaging Inc., Pittsburgh, PA) on a Macintosh computer. In most cases, a projection image (max density or sum) was composed from a stack of images at relevant focus levels using the public domain NIH Image program (developed at the U.S. National Institutes of Health; available at http://rsb.info.nih.gov/nih-image/ (accessed 11.02. 2013)).

For testing the computer-assisted cell counting method, images were chosen by the following criteria: retinas from different species, differences in quality concerning morphology (different amount of cell debris, broken cones etc.) and contrast between labeled cells and background (see Table 1). Images with additional labeled structures like horizontal cells or blood vessels have been analyzed as well. The respective objects of interest were then manually counted by an experienced coauthor (C.S.), and the results were tabulated for comparison with the outcome from the automatic counting method (see below). 
Table 1 Retinal image data

\begin{tabular}{|c|c|c|c|c|c|c|c|c|c|c|}
\hline No. & Species & Eye & Retina region & $\begin{array}{l}\text { Labeled } \\
\text { structures }\end{array}$ & Staining & $\begin{array}{l}\text { Density per } \\
\mathrm{mm}^{2}\end{array}$ & File name & Image size & $\begin{array}{l}\text { Remarks about image } \\
\text { quality and particularities }\end{array}$ & $\begin{array}{l}\text { Shown in } \\
\text { Figure(s) }\end{array}$ \\
\hline 1 & Orangutan & Left & $\begin{array}{l}\text { Sup. periphery, } \\
\text { between } 10 \mathrm{~mm} \text { from } \\
\text { fovea and ora serrata }\end{array}$ & $\begin{array}{l}\text { S-cones, } \\
\text { blood vessels }\end{array}$ & $\begin{array}{l}\text { JH455/DAB, } \\
\text { Collagen IV/DAB }\end{array}$ & 190 (cones) & OrangWhM & $1024 \times 854$ & $\begin{array}{l}\text { Sufficient quality, } \\
\text { but vessels present }\end{array}$ & $1 \mathrm{~A}$ \\
\hline 2 & Orangutan & Left & $\begin{array}{l}\text { Inf. periphery, } \\
\text { between } 11 \mathrm{~mm} \text { from } \\
\text { fovea and ora serrata }\end{array}$ & $\begin{array}{l}\text { S-cones, } \\
\text { blood vessels }\end{array}$ & $\begin{array}{l}\text { JH455/DAB, } \\
\text { Collagen IV/DAB }\end{array}$ & 156 (cones) & OrUinf-0001pr & $1026 \times 854$ & $\begin{array}{l}\text { Sufficient quality, } \\
\text { but vessels present }\end{array}$ & $4 \mid-J$ \\
\hline 3 & Domestic cat & & & S-cones & $J H 455 / D A B$ & 860 & loStack & $1024 \times 1024$ & $\begin{array}{l}\text { Sufficient quality and contrast, } \\
\text { but small cones }\end{array}$ & \\
\hline 4 & Domestic cat & & Inf. retina & S-cones & $J H 455 / D A B$ & 851 & luStack & $1024 \times 1024$ & $\begin{array}{l}\text { Sufficient quality and contrast, } \\
\text { but small cones }\end{array}$ & $1 \mathrm{~B}$ \\
\hline 5 & Domestic cat & & & S-cones & $\mathrm{JH} 455 / \mathrm{DAB}$ & 827 & roStack & $1024 \times 1024$ & $\begin{array}{l}\text { Sufficient quality and contrast, } \\
\text { but small cones }\end{array}$ & \\
\hline 6 & Domestic cat & & & S-cones & $\mathrm{JH} 455 / \mathrm{DAB}$ & 904 & ruStack & $1024 \times 1024$ & $\begin{array}{l}\text { Sufficient quality and contrast, } \\
\text { but small cones }\end{array}$ & \\
\hline 7 & Manul & & & S-cones & $J H 455 / D A B$ & 195 & bcergcomp & $1024 \times 1024$ & $\begin{array}{l}\text { Sufficient quality and contrast, } \\
\text { but small cones }\end{array}$ & \\
\hline 8 & Eurasian Lynx & Left & Periphery & S-cones & $J H 455 / D A B$ & 1076 & Movie-17-1 & $1024 \times 1024$ & Good quality and contrast & $4 A-B$ \\
\hline 9 & Cheetah & & & S-cones & $\mathrm{JH} 455 / \mathrm{DAB}$ & 130 & $1+5$ CSCStack & $1024 \times 1024$ & Good quality and contrast & \\
\hline 10 & Cheetah & & & S-cones & $J H 455 / D A B$ & 1055 & Stack SCFoc. & $1024 \times 1024$ & Good quality and contrast & \\
\hline 11 & Jaguar & Right & Periphery & $\begin{array}{l}\text { S-cones, } \\
\text { horizontal cells }\end{array}$ & $\mathrm{JH} 455 / \mathrm{DAB}$ & 440 (cones) & Combination & $1024 \times 1024$ & $\begin{array}{l}\text { Good quality, but additional } \\
\text { cell type present }\end{array}$ & $1 \mathrm{C}, 4 \mathrm{G}-\mathrm{H}$ \\
\hline 12 & Pangolin & Right & Mid periphery & M-/L-cones & $\mathrm{JH} 492 / \mathrm{DAB}$ & 2290 & 492_20_09 & $1024 \times 1024$ & Background very blotchy & $\begin{array}{l}1 \mathrm{D}, 2 \mathrm{~A}-\mathrm{F} \\
4 \mathrm{C}-\mathrm{D}\end{array}$ \\
\hline 13 & Agouti & Left & $\begin{array}{l}\text { Sup. temp. } \\
\text { periphery }\end{array}$ & S-cones & $\mathrm{JH} 455 / \mathrm{DAB}$ & 705 & A4scal & $1024 \times 853$ & Low contrast, cell debris present & $1 \mathrm{E}$ \\
\hline 14 & Agouti & & & S-cones & $\mathrm{JH} 455 / \mathrm{DAB}$ & 790 & A5scal & $1024 \times 853$ & Low contrast, cell debris present & \\
\hline 15 & Agouti & & & S-cones & $\mathrm{JH} 455 / \mathrm{DAB}$ & 855 & A6scal & $1024 \times 853$ & Low contrast, cell debris present & \\
\hline 16 & Agouti & left & Temp. periphery & S-cones & $J H 455 / D A B$ & 480 & B1scal & $1024 \times 853$ & Poor quality, cell debris present & $1 F, 4 E-F$ \\
\hline 17 & Agouti & & & S-cones & $J H 455 / D A B$ & 905 & B2scal & $1024 \times 853$ & Poor quality, cell debris present & \\
\hline 18 & Agouti & & & S-cones & $\mathrm{JH} 455 / \mathrm{DAB}$ & 3500 & C4scal & $1024 \times 853$ & Poor quality, cell debris present & \\
\hline
\end{tabular}



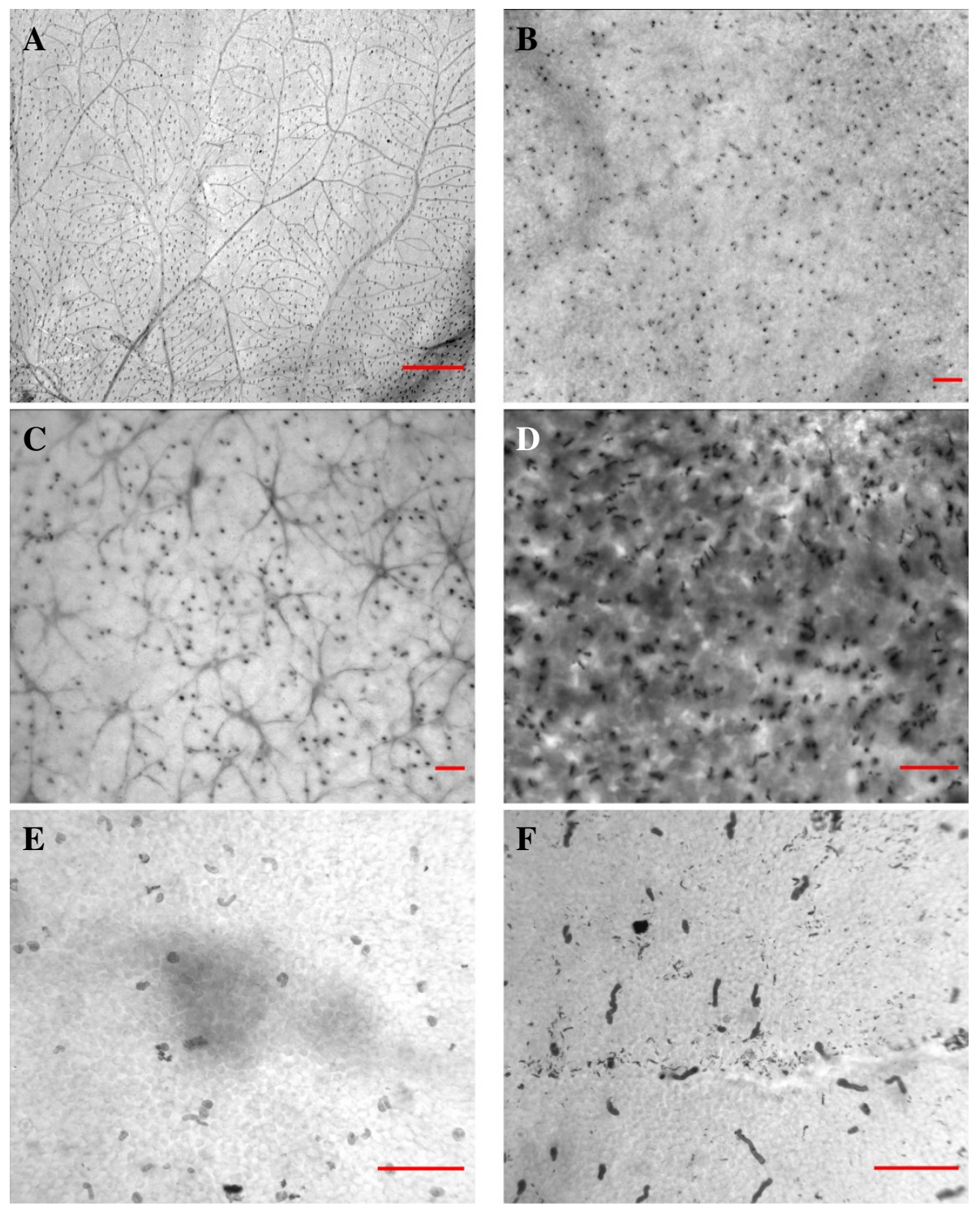

Figure 1 Examples of retinal micrographs used in the experiments. The scale, indicated by the red bar, is $500 \mu \mathrm{m}$ in (A) and $50 \mu \mathrm{m}$ in (B)-(F). (A) Orangutan, No. 1, S-cones and vessels labeled. (B) Domestic cat, No. 4 (clip), S-cones labeled. (C) Jaguar, No. 11 (clip), S-cones and horizontal cells labeled. (D) Pangolin, No. 12 (clip), M-/L-cones labeled. (E) Agouti, No. 13, S-cones labeled. (F) Agouti, No. 16, S-cones labeled. For more details, see Table 1 .

\section{Description of the detection and counting method}

Due to the reasons mentioned in the introduction, the immediate segmentation of the retina image data $I^{(0)}$ by intensity thresholding leads in many instances to poor results, see below. Therefore, we carry out two processing steps before segmentation. In a first step, we generate from the original image $I^{(0)}$, cf. Figure 2A, a medianfiltered version $\widetilde{I}$ using window size $m$ and subtract it from $I^{(0)}$, which results in a considerable removal of brightness fluctuations in the retinal background, cf. Figure 2B. Subsequently, we subject the image $I^{(0)}-\widetilde{I}=I^{(1)}$ to a Rudin-Osher-Fatemi TV denoising procedure, cf. [11].
This method, representing a well-established standard in mathematical image processing, may be understood as a kind of filtering, which generates a coarsened, cartoonlike version of the input data, cf. Figure $2 \mathrm{C}$. Nevertheless, during this procedure the images of the dyed retinal cells will be conserved as spots. In mathematical terms, TV denoising means to solve an continuous optimization problem, namely

$$
F(x)=\int_{\Omega}\left(x(s)-I^{(1)}(s)\right)^{2} d s+\frac{\alpha}{2}|x|_{T V} \longrightarrow \text { Min } !
$$


for an unknown function $x(s): \Omega \rightarrow[0,1]$, which represents the converted ("filtered") image. For more details, we refer to the Appendix. Let us only remark that the number $\alpha>0$ within (1) remains fixed from the outset. For the numerical solution of problem (1), surprisingly efficient methods are available by now. In our present study, a recently published solver (by Chambolle/Pock, cf. [15]) has been implemented as a MATLAB subroutine. The segmentation of the output $x=\left(x_{i j}\right)$ of the TV denoising step will be performed now by application of the following rule: After calculating the expectation $E(x)$ and variance $\operatorname{Var}(x)$, we declare all pixels $x_{i j}$ with

$$
x_{i j}<E(x)-c \cdot \operatorname{Var}(x)
$$

as "black enough" to belong to images of photoreceptor cells. Finally, all "black" features consisting of a number of connected adjacent pixels, which is bigger than a given threshold size $f$, are automatically counted, cf. Figure 2D.

In this method, no more than three parameters remain to be adjusted manually. These are: the window size $m$ for the median filter, the parameter $c$ in (2), which influences the contrast differentiation between photoreceptor cells and the background, and the minimal size $f$ of a connected feature to be recognized as a photoreceptor cell.

Our algorithm can be summarized as follows:

\section{Implementation}

\section{Implementation as a MATLAB tool}

Algorithm 1 has been implemented as a MATLAB tool with a graphical user interface (cf. Figure 3A), which allows for batch processing of multiple images. It has been tested on MATLAB 7.14.0.739 (R2012a) and requires the MATLAB Image Processing Toolbox (documented at http://www.mathworks.com/products/ matlab and http://www.mathworks.com/products/image (accessed 11.02.2013)). In the following, details regarding the implementation of the procedure are given. In Step 1, the background homogenization, the median filtering is realized in a straightforward manner by calling the MATLAB procedure medfilt2(image, $[\mathrm{m}, \mathrm{m}]$, 'symmetric') which is part of the image processing toolbox. For the TV denoising in Step 2, the primal-dual algorithm from [15] is utilized. It is realized by performing $N$ steps of the iteration

$$
\left\{\begin{array}{c}
\left(\begin{array}{l}
p^{(1), k+1} \\
p^{(2), k+1}
\end{array}\right)=P_{\alpha}\left(\left(\begin{array}{l}
p^{(1), k} \\
p^{(2), k}
\end{array}\right)+\tau_{d}\left(\begin{array}{c}
\partial_{s_{1}}^{+} \bar{x}^{k} \\
\partial_{s_{2}}^{+} \bar{x}^{k}
\end{array}\right)\right), P_{\alpha}(v)_{i j} \\
=\frac{\alpha}{\operatorname{Max}\left(\alpha, \sqrt{\left(v_{i j}^{(1)}\right)^{2}+\left(v_{i j}^{(2)}\right)^{2}}\right)}\left(\begin{array}{c}
v_{i j}^{(1)} \\
v_{i j}^{(2)}
\end{array}\right) \\
x^{k+1}=\frac{1}{1+\tau_{p}} x^{k}+\frac{\tau_{p}}{1+\tau_{p}}\left(I^{(1)}+\partial_{s_{1}}^{-} p^{(1), k+1}+\partial_{s_{2}}^{-} p^{(2), k+1}\right) \\
\bar{x}^{k+1}=2 x^{k+1}-\bar{x}^{k}
\end{array}\right.
$$

Algorithm 1 Automatic segmentation after TV denoising

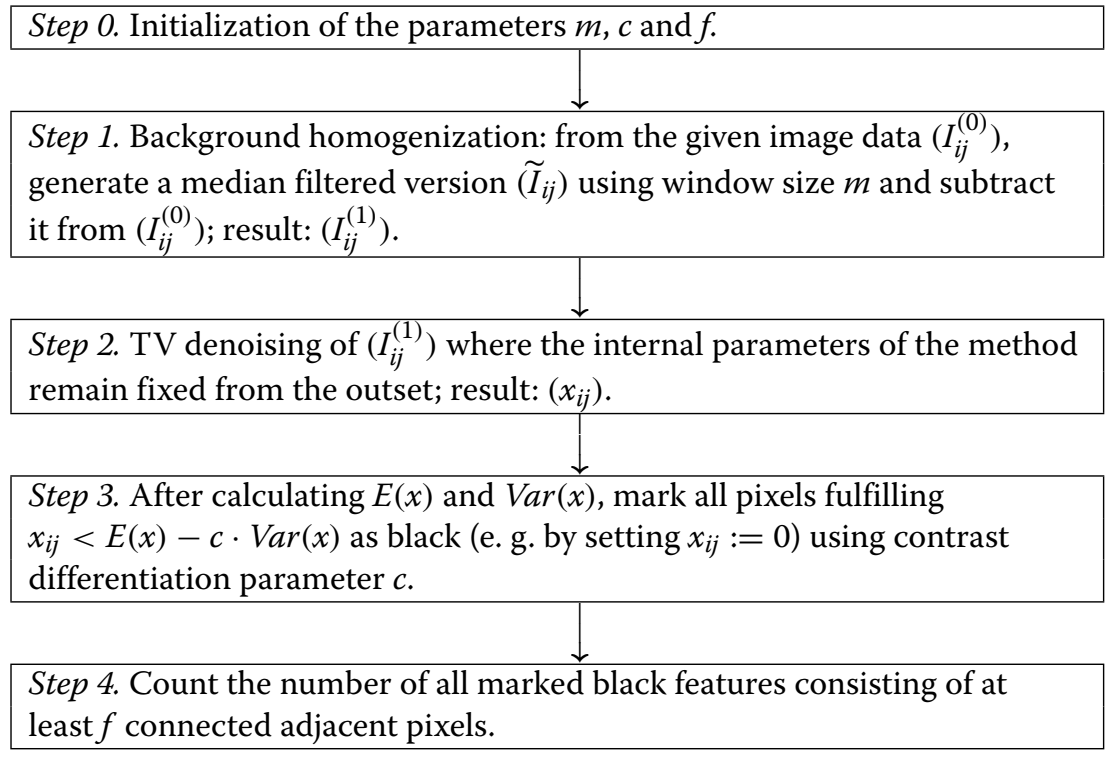


with step sizes $\tau_{p}, \tau_{d}>0$ such that $\tau_{p} \cdot \tau_{d} \leq 0.125$ and the forward and backward finite difference operators

$$
\begin{aligned}
& \left(\partial_{s_{1}}^{+} \bar{x}^{k}\right)_{i j}=\left\{\begin{array}{c}
\bar{x}_{i+1, j}^{k}-\bar{x}_{i j}^{k} \text { if } 1 \leq i<n, \\
0 \text { else },
\end{array}\right. \\
& =\left\{\begin{array}{cc}
\bar{x}_{i, j+1}^{k}-\bar{x}_{i j}^{k} \text { if } 1 \leq j<r, \\
0 \text { else, }
\end{array}\right. \\
& \left(\partial_{s_{1}}^{-} p^{(1), k+1}\right)_{i j}=\left\{\begin{array}{cc}
p_{1 j}^{(1), k+1} & \text { if } i=1, \\
p_{i j}^{(1), k+1}-p_{i-1, j}^{(1), k+1} & \text { if } 1<i<n, \\
-p_{n-1, j}^{(1), k+1} & \text { if } i=n,
\end{array}\right.
\end{aligned}
$$

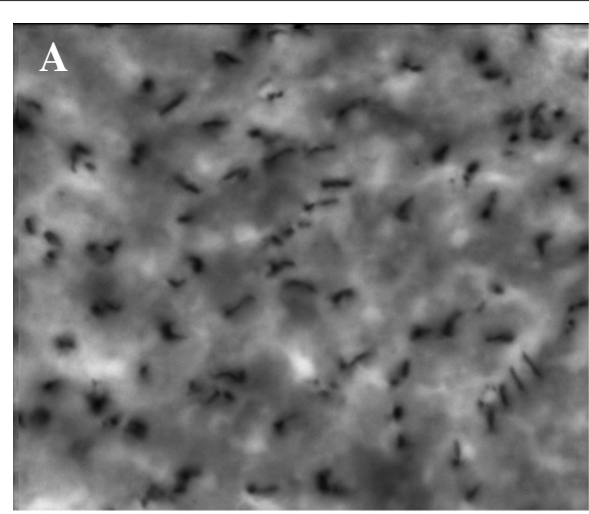

$$
\left(\partial_{s_{2}}^{-} p^{(2), k+1}\right)_{i j}=\left\{\begin{array}{cl}
p_{i 1}^{(2), k+1} & \text { if } j=1, \\
p_{i j}^{(2), k+1}-p_{i, j-1}^{(2), k+1} & \text { if } 1<j<r, \\
-p_{i, r-1}^{(2), k+1} & \text { if } j=r .
\end{array}\right.
$$

The initializations are $x^{0}=\bar{x}^{0}=I^{(1)}, p^{(1), 0}=p^{(2), 0}=0$, and the output will be given by $\left(x_{i j}\right)=\left(x_{i j}^{N}\right)$. As default values of the regularization parameter and the number of iterations, we use $\alpha=0.05$ and $N=50$. Note that, in principle, other minimization algorithms could be implemented for solving the TV denoising problem. For

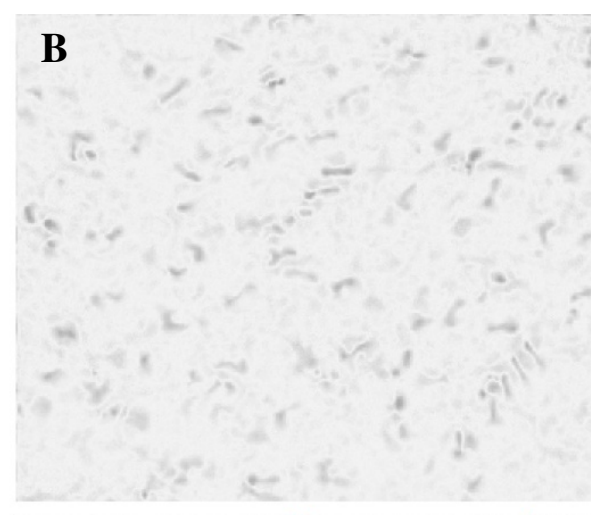

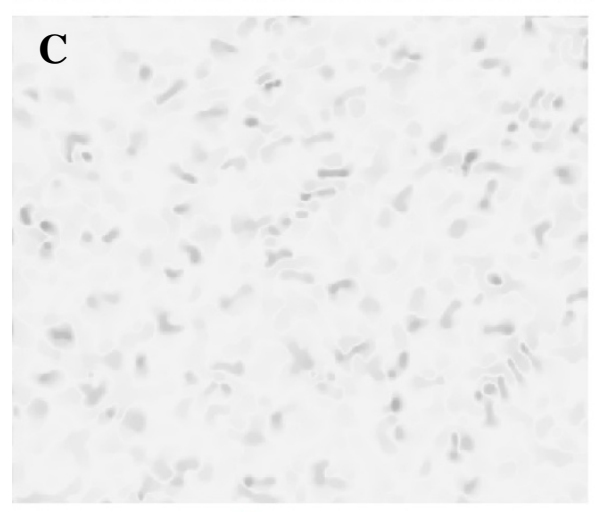
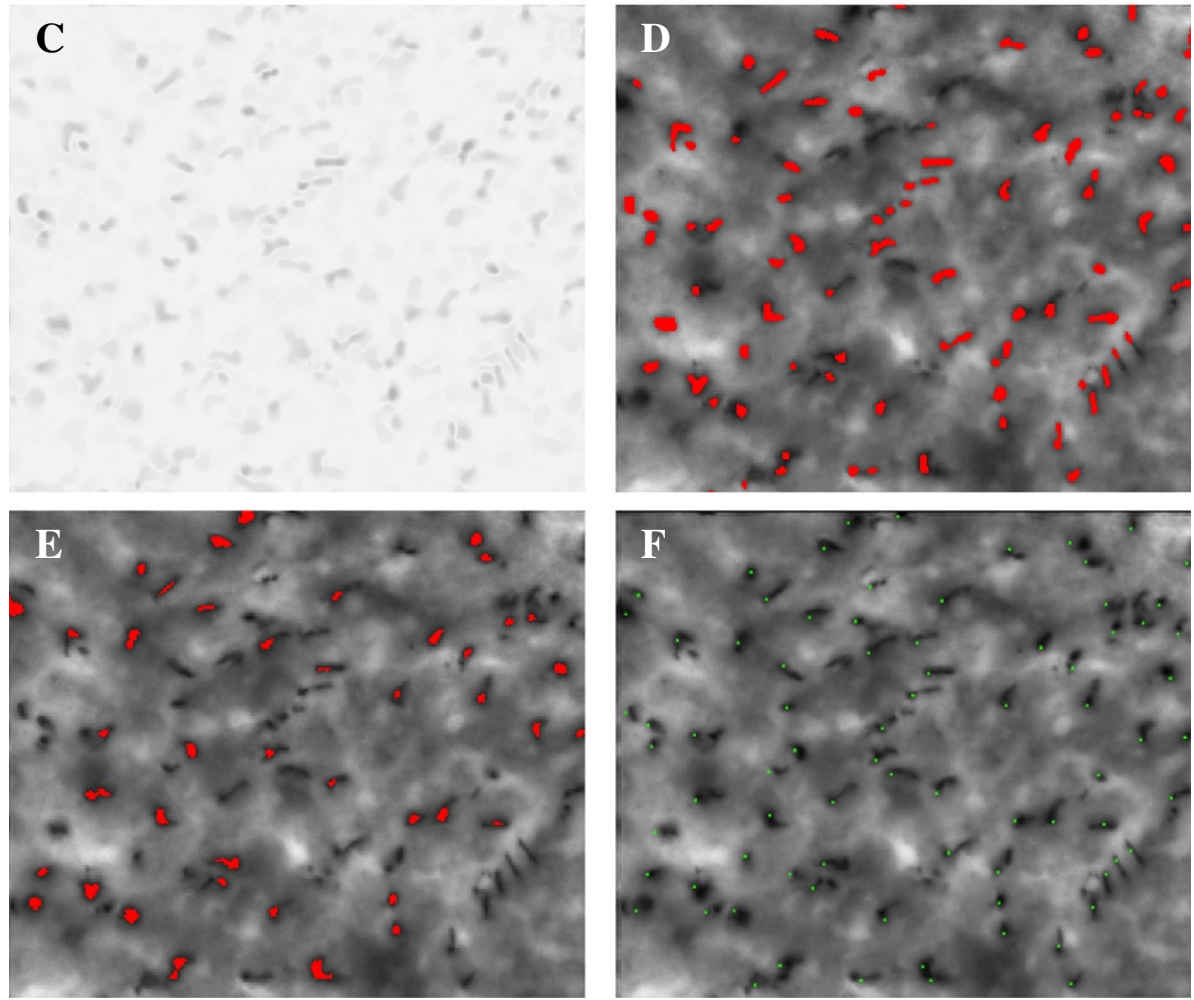

Figure 2 The counting procedure. (A) Original data (Pangolin, No. 12, upper left part). (B) Output after Step 1 (subtraction of median), grey values divided by factor 1.05. (C) Output of TV denoising after Step 2, grey values divided by factor 1.05. (D) Output of Step 4, superimposed to the original image (counted features dyed in red color). (E) Result of direct segmentation, superimposed to the original image (counted features dyed in red color). (F) Result of manual count (counted cells tagged with green dots). 

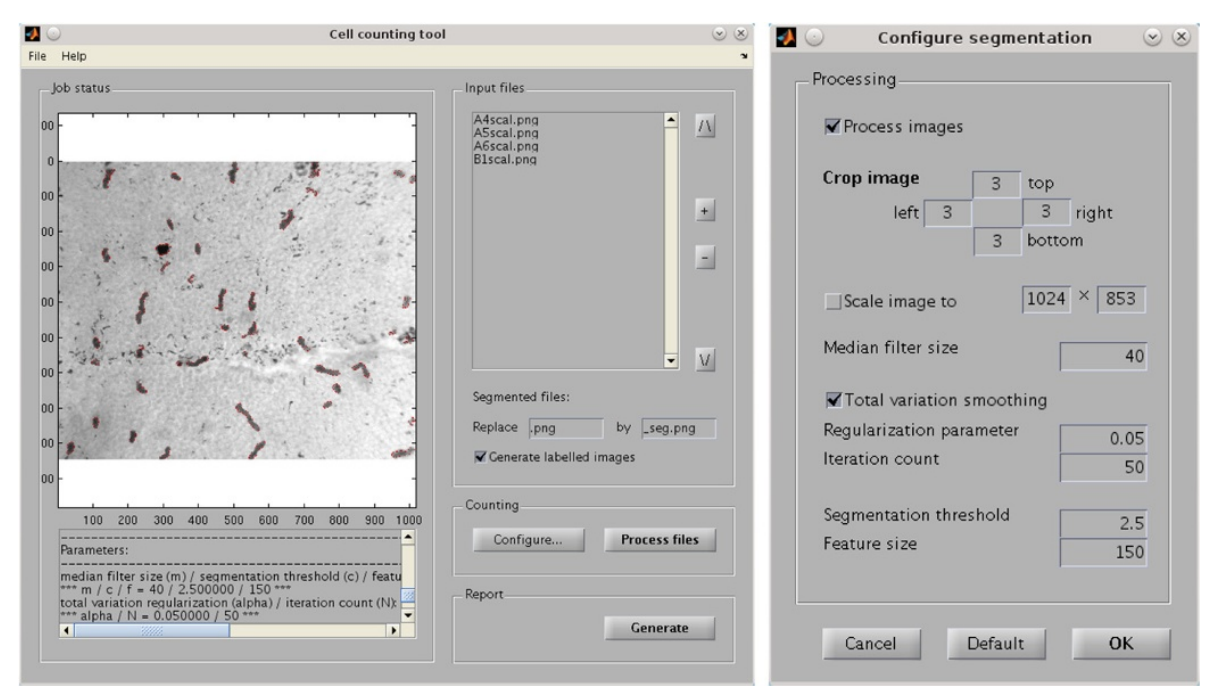

Figure 3 Screenshots of the MATLAB software tool. (A) Main graphical user interface. (B)

(B) The configuration dialog.

instance, we mention the generalized TV approach from [16] and the optimal control method described in [17], which is based on the interior-point solver IPOPT $[18,19]$.

The thresholding in Step 3 is realized with the help of the built-in MATLAB functions mean and var. Finally, for Step 4, the labelling and counting procedure, the function bwlabel is utilized, which is again part of the image processing toolbox. It yields a labelled image in which each connected component is identified by a positive integer. With this information, the identification and counting of those connected components, which comprise at least $f$ pixels, can be easily realized.

\section{Usage}

Usually, in order to analyze the topography of items in a retina preparation, a considerable number of image files has to be generated, each showing a segment. Our software tool was especially designed to cope with multiple files showing similar structures. In this situation, it is possible to start with a manual count within one or two typical images in order to calibrate the parameters $m, c$ and $f$. This can be done by starting the program and adding a single image to the file list. In most cases, the default parameters give a good starting point, hence one can perform a segmentation in order to decide whether one is satisfied with the results. Otherwise, adjust the parameters $m$, $c$ and $f$ using the configuration dialog (cf. Figure 3B) and try again. Once the parameters have been adjusted, they can be utilized for the analysis of the whole image set. The batch processing feature of the software allows to perform this analysis without further user interaction: simply add the remaining files to the list and start the segmentation procedure. Finally, a report which lists, for each file in the batch, the number and mean density of detected cells as well as their positions, can be automatically generated. As default values for the parameters $m, c$ and $f$, we encoded $m=30, c=2.5$ and $f=5$. Eventually, for convenience and reproducibility, the tool also provides saving and loading of the file list as well as of a list of all parameters.

\section{Results and discussion}

The results of automatical counting are documented in Tables 2, 3 and 4. In order to justify the application of a counting procedure, it is essential that manually counted data are reliably matched. Consequently, the counts generated by TV denoising and subsequent segmentation are compared with carefully realized manual counts. The results are listed in Table 2. Furthermore, for every automatic count the number of recognized cells, which have also been marked in the manual counting procedure (Table 3), as well as the number of artifacts (Table 4) were identified. For comparison, we performed automatic counts by direct segmentation without preceding TV denoising using the same parameters $c$ and $f$ as in Algorithm 1.

The quality requirements for automatic counts depend on the specific goal of interest. When assessing general distribution patterns or gradients for certain types of receptors, a relative error of $10 \%$ may be acceptable while for the analysis of degeneration or proliferation phenomena or the detection of initial points of density changes, an error of about $5 \%$ and less is desirable. Table 2 shows that the latter goal has been realized by the TV/segmentation method in 12 of 18 cases while the error is below $10 \%$ in 16 of 18 cases. The mean error amounts to 5.9\%. Moreover, Table 3 shows that the method recognizes correctly more 
Table 2 Overall cell counts by different methods

\begin{tabular}{|c|c|c|c|c|c|}
\hline No. & Image & Manual count \# & $\begin{array}{l}\text { Direct segm. } \\
\text { \# / rel. error }\end{array}$ & $\begin{array}{l}\text { TV denoising \& segm. } \\
\text { \# / rel. error }\end{array}$ & $m / c / f$ \\
\hline & Orangutan & & & & \\
\hline 1 & OrangWhM & 2328 & 1784 / 24.9 & $2286 / 1.8$ & $30 / 2.0 / 5$ \\
\hline \multirow[t]{2}{*}{2} & OrUinf-0001pr & 1192 & 793 / 33.5 & $1168 / 2.0$ & $30 / 3.0 / 5$ \\
\hline & Domestic cat & & & & \\
\hline 3 & loStack & 557 & $518 / 7.0$ & $543 / 2.5$ & $30 / 2.5 / 5$ \\
\hline 4 & luStack & 552 & $584 / 5.8$ & $562 / 1.8$ & $30 / 2.5 / 5$ \\
\hline 5 & roStack & 536 & $539 / 0.6$ & $520 / 3.0$ & $30 / 2.5 / 5$ \\
\hline \multirow[t]{2}{*}{6} & ruStack & 584 & $573 / 1.9$ & $573 / 1.9$ & $30 / 2.5 / 5$ \\
\hline & Manul & & & & \\
\hline \multirow[t]{2}{*}{7} & bcergcomp & 128 & $110 / 14.1$ & $128 / 0.0$ & $20 / 2.5 / 25$ \\
\hline & Lynx & & & & \\
\hline \multirow[t]{2}{*}{8} & Movie-17-1 & 708 & 675 / 4.7 & 659 / 6.9 & $30 / 2.5 / 5$ \\
\hline & Cheetah & & & & \\
\hline 9 & $1+5$ CSCStack & 85 & $84 / 1.2$ & $91 / 7.1$ & $30 / 3.5 / 40$ \\
\hline \multirow[t]{2}{*}{10} & Stack SCFoc. & 687 & $656 / 4.5$ & $634 / 7.7$ & $30 / 2.5 / 5$ \\
\hline & Jaguar & & & & \\
\hline \multirow[t]{2}{*}{11} & Combination & 289 (S-cones) & $177 / 38.8$ & $275 / 4.8$ & $30 / 3.5 / 5$ \\
\hline & Pangolin & & & & \\
\hline \multirow[t]{2}{*}{12} & 492_20_09 & 369 & $109 / 70.5$ & $367 / 0.5$ & $20 / 2.5 / 25$ \\
\hline & Agouti & & & & \\
\hline 13 & A4scal & 43 & $14 / 67.4$ & $44 / 2.3$ & $40 / 2.5 / 150$ \\
\hline 14 & A5scal & 48 & $20 / 58.3$ & $54 / 12.5$ & $40 / 2.5 / 150$ \\
\hline 15 & A6scal & 52 & $44 / 15.4$ & $53 / 1.9$ & $40 / 2.5 / 150$ \\
\hline 16 & B1scal & 31 & 35 / 12.9 & 43 / 38.7 & $40 / 2.5 / 150$ \\
\hline 17 & B2scal & 55 & $51 / 7.3$ & $56 / 1.8$ & $40 / 2.5 / 150$ \\
\hline \multirow[t]{3}{*}{18} & C4scal & $213(?)$ & $95 / 55.4$ & 234 / 9.8 & $30 / 2.5 / 50$ \\
\hline & Mean error & & 23.6 & 5.9 & \\
\hline & Standard dev. & & 23.6 & 8.6 & \\
\hline
\end{tabular}

Within Algorithm 1, the following parameters have been chosen: The median is taken over a field of $m \times m$ pixels, the TV denoising procedure is applied with regularization parameter $\alpha=0.05$ and 50 iterations, the contrast differentiation parameter is $c$, and the minimal size of a recognized feature is $f$ pixels. For comparison, a direct segmentation of the images has been performed as well, applying Steps 3 and 4 of Algorithm 1 with the same values of $c$ and $f$ directly to the image data. Together with the automatic counts, their accuracy is given in terms of the relative error (in percents) related to the manual counts from the third column.

than $90 \%$ of the manually marked cells in 15 of 18 cases (93.0\% in the mean). The number of artifacts contained in the counts, as listed in Table 4 , amounts to $8.7 \%$ in the mean. Thus, given that the $\mathrm{TV} /$ segmentation method makes no use of additional information about the shape of the cells or the variation of their size, these results are quite satisfactory.

As to be expected, our results show that automatic counting by the $\mathrm{TV} /$ segmentation method is superior to direct segmentation in every respect. The mean relative error produced by the latter amounts to as much as $23.6 \%$, and the relative error goes below $10 \%$ in 8 of 18 cases only. The loss of precision is mostly caused by the fact that, by direct segmentation, a significantly smaller number of marked cells is recognized than by the TV/segmentation method (cf. Figure 2D-F) while the ratio of artifacts produced by both methods is comparable. The superiority of the TV/segmentation method can be seen as well by comparing the standard deviations of the indicators. Let us remark that, during our experiments, we further observed that the $\mathrm{TV} /$ segmentation method is even superior to direct segmentation after subtraction of the median (Steps 1, 3 and 4 of Algorithm 1).

As the patterns within the Orangutan retina closely resemble the organization within the human one, similar results are to be expected for human samples. 
Table 3 Number of correctly recognized cells within the automatic counts

\begin{tabular}{|c|c|c|c|c|c|}
\hline No. & Image \# & Manual count & $\begin{array}{l}\text { Direct segm. } \\
\text { \# / percent }\end{array}$ & $\begin{array}{l}\text { TV denoising \& segm. } \\
\text { \# / percent }\end{array}$ & $m / c / f$ \\
\hline & Orangutan & & & & \\
\hline 1 & OrangWhM & 2328 & $1652 / 71.0$ & $2176 / 93.4$ & $30 / 2.0 / 5$ \\
\hline \multirow[t]{2}{*}{2} & OrUinf-0001pr & 1192 & 735 / 61.7 & 1077 / 90.4 & $30 / 3.0 / 5$ \\
\hline & Domestic cat & & & & \\
\hline 3 & loStack & 557 & 474 / 85.1 & $512 / 91.9$ & $30 / 2.5 / 5$ \\
\hline 4 & luStack & 552 & $531 / 96.2$ & 527 / 95.5 & $30 / 2.5 / 5$ \\
\hline 5 & roStack & 536 & $502 / 93.6$ & 499 / 93.1 & $30 / 2.5 / 5$ \\
\hline \multirow[t]{2}{*}{6} & ruStack & 584 & $530 / 90.8$ & $545 / 93.3$ & $30 / 2.5 / 5$ \\
\hline & Manul & & & & \\
\hline \multirow[t]{2}{*}{7} & bcergcomp & 128 & $80 / 62.5$ & $126 / 98.4$ & $20 / 2.5 / 25$ \\
\hline & Lynx & & & & \\
\hline \multirow[t]{2}{*}{8} & Movie-17-1 & 708 & 642 / 90.7 & $640 / 90.4$ & $30 / 2.5 / 5$ \\
\hline & Cheetah & & & & \\
\hline 9 & $1+5$ CsCStack & 85 & $81 / 95.3$ & $85 / 100.0$ & $30 / 3.5 / 40$ \\
\hline \multirow[t]{2}{*}{10} & Stack SCFoc. & 687 & 637 / 92.7 & $624 / 90.8$ & $30 / 2.5 / 5$ \\
\hline & Jaguar & & & & \\
\hline \multirow[t]{2}{*}{11} & Combination & 289 (cones) & 164 / 56.7 & $253 / 87.5$ & $30 / 3.5 / 5$ \\
\hline & Pangolin & & & & \\
\hline \multirow[t]{2}{*}{12} & 492_20_09 & 369 & 104 / 28.2 & $297 / 80.5$ & $20 / 2.5 / 25$ \\
\hline & Agouti & & & & \\
\hline 13 & A4scal & 43 & $12 / 27.9$ & $40 / 93.0$ & $40 / 2.5 / 150$ \\
\hline 14 & A5scal & 48 & $16 / 33.3$ & 45 / 93.8 & $40 / 2.5 / 150$ \\
\hline 15 & A6scal & 52 & $40 / 76.9$ & $50 / 96.2$ & $40 / 2.5 / 150$ \\
\hline 16 & B1scal & 31 & $29 / 93.5$ & $31 / 100.0$ & $40 / 2.5 / 150$ \\
\hline 17 & B2scal & 55 & $50 / 90.9$ & 54 / 98.2 & $40 / 2.5 / 150$ \\
\hline \multirow[t]{3}{*}{18} & C4scal & $213(?)$ & $77 / 36.1$ & $185 / 86.8$ & $30 / 2.5 / 50$ \\
\hline & Mean percentage & & 71.3 & 93.0 & \\
\hline & Standard dev. & & 24.5 & 4.8 & \\
\hline
\end{tabular}

The percentages are given in relation to the manual counts in the third column.

Let us briefly compare the proposed method with other approaches pursued in the literature. In [8], the authors perform the image processing steps by use of a commercial software package which, unfortunately, comes as a "black box" without documentation of the internally utilized algorithms. In $[9,10,20,21]$, after certain presmoothing/denoising steps, watershed segmentation is employed. Additionally, before segmenting, in [20] an illumination correction is performed while the authors in [10] interpose a contrast enhancement step. A common feature of all approaches is the necessity to select a number of parameters, including the (expected or minimal) feature size, by the experimenter.

Although preprocessing steps, particularly denoising or smoothing of the raw image data, are crucial for the quality of the results of subsequent segmentation, they have not been thoroughly documented in the cited references (if at all), and their dependence on additional, manually tuned parameters remains unclear. Moreover, any denoising method generates artifacts, thus modifying fine structures within the images in a specific way. In contrast to this situation, the preprocessing steps involved in our method (median filtering and TV denoising) are traceable and reproducible, including the manual setting of the single parameter $m$. For the TV denoising method, the characteristic artifacts are well-investigated (see e. g. the discussion in [16], pp. 519 ff.). In fact, this method has been deliberately selected in order to take advantage of its well-known "cartooning" effect. As a further difference, we segment by intensity thresholding 
Table 4 Artifacts within the automatic counts produced by the different methods

\begin{tabular}{|c|c|c|c|c|}
\hline No. & Image & $\begin{array}{l}\text { Direct segm. } \\
\text { \# / percent }\end{array}$ & $\begin{array}{l}\text { TV denoising \& segm. } \\
\text { \# / percent }\end{array}$ & $m / c / f$ \\
\hline & Orangutan & & & \\
\hline 1 & OrangWhM & $132 / 7.4$ & $110 / 4.8$ & $30 / 2.0 / 5$ \\
\hline \multirow[t]{2}{*}{2} & OrUinf-0001pr & $58 / 7.3$ & $91 / 7.8$ & $30 / 3.0 / 5$ \\
\hline & Domestic cat & & & \\
\hline 3 & loStack & $44 / 8.5$ & $31 / 5.7$ & $30 / 2.5 / 5$ \\
\hline 4 & luStack & $53 / 9.1$ & $35 / 6.2$ & $30 / 2.5 / 5$ \\
\hline 5 & roStack & $37 / 6.9$ & $21 / 4.0$ & $30 / 2.5 / 5$ \\
\hline \multirow[t]{2}{*}{6} & ruStack & $43 / 7.5$ & $28 / 4.9$ & $30 / 2.5 / 5$ \\
\hline & Manul & & & \\
\hline \multirow[t]{2}{*}{7} & bcergcomp & $30 / 23.4$ & $2 / 1.6$ & $20 / 2.5 / 25$ \\
\hline & Lynx & & & \\
\hline \multirow[t]{2}{*}{8} & Movie-17-1 & $33 / 4.9$ & $19 / 2.9$ & $30 / 2.5 / 5$ \\
\hline & Cheetah & & & \\
\hline 9 & $1+5$ CSCStack & $3 / 3.6$ & $6 / 6.6$ & $30 / 3.5 / 40$ \\
\hline \multirow[t]{2}{*}{10} & Stack SCFoc. & $19 / 2.9$ & $10 / 1.6$ & $30 / 2.5 / 5$ \\
\hline & Jaguar & & & \\
\hline \multirow[t]{2}{*}{11} & Combination & $13 / 7.3$ & $22 / 8.0$ & $30 / 3.5 / 5$ \\
\hline & Pangolin & & & \\
\hline \multirow[t]{2}{*}{12} & 492_20_09 & $5 / 4.6$ & $70 / 19.1$ & $20 / 2.5 / 25$ \\
\hline & Agouti & & & \\
\hline 13 & A4scal & $2 / 14.3$ & $4 / 9.1$ & $40 / 2.5 / 150$ \\
\hline 14 & A5scal & $4 / 20.0$ & $9 / 16.7$ & $40 / 2.5 / 150$ \\
\hline 15 & A6scal & $4 / 9.1$ & $3 / 5.7$ & $40 / 2.5 / 150$ \\
\hline 16 & B1scal & $6 / 17.1$ & $12 / 27.9$ & $40 / 2.5 / 150$ \\
\hline 17 & B2scal & $1 / 2.0$ & $2 / 3.6$ & $40 / 2.5 / 150$ \\
\hline \multirow[t]{3}{*}{18} & C4scal & $18 / 18.9$ & $49 / 20.9$ & $30 / 2.5 / 50$ \\
\hline & Mean percentage & 9.7 & 8.7 & \\
\hline & Standard dev. & 6.1 & 7.2 & \\
\hline
\end{tabular}

The percentages are given in relation to the corresponding counts from columns 4 and 5 in Table 2 .

instead of watershedding. The latter approach is well suited for the analysis of large, clumpy cell aggregates while our method is better suited for the detection of single cells to be differentiated from a more or less blotchy background, which may contain additional structures like vessels or different cell types. The segmentation depends on no more than two further parameters $c$ and $f$, which have to be selected on the base of the observed contrast as well as of a reasonable guess of the feature size. A possible improvement could be the introduction of an additional upper bound for the size of the recognized features, thus reducing and possibly underestimating the number of cells since larger aggregates formed of merged cell images will then be excluded.
The reliability of our method, when evaluated by the mean relative error of automatic counting in relation to manual counts (5.9\% as documented above), fits well within the range of errors documented in the cited references: [9], p. 1969, Figure one: $\leq 10 \%$ in 11 of 23 cases; [10], p. 641, Figure two: $\leq 5 \%$ in 31 of 40 cases; [20], p. R100.7, Figure two(A): $6 \%$ and $17 \%$; [21], p. 589, Figure three: $\leq 10 \%$ in roughly half of the cases.

Due to its traceability, the TV denoising method offers the further advantage of easy reimplementation. On the one hand, this is possible with respect to the use of non-proprietary software, on the other hand, the method may be carried over (after optimization of the code as necessary) to the analysis of three- or multidimensional 
data stacks. Concerning the runtime behaviour, the analysis of an $1024 \times 1024-$ pixel image takes typically less than $40 \mathrm{sec}$ (on a Mini-PC equipped with four processors Intel(R)Core(TM)i3 CPU M380@2.53 GHz) where approximately half of the time is consumed by the median filtering procedure. No particular attempts for tuning have been made.

The limitations of the proposed method are exemplified in Figure 4. It shows typical errors within the automatic counting, which would be avoided by a human examiner. Adjacent cells are merged and counted as a single feature (Figure 4A and B). Another typical error occurs when a single photoreceptor cell does not lie exactly in the image plane. The resulting blurred image, may be "broken" into two or more features, resulting in double or multiple counts (Figure 4C and D). Also, cell debris and background spots may not be recognized as such (Figure 4E and F). Consequently, for heavily contaminated samples such as Nos. 16 and 18, a reduction of the quality of the automatic count is to be expected (in Table 2, No. 16 is the outlier). If background structures such as horizontal cells or vessels are present in the samples, it may happen that parts of them will be counted for cells as well (Figure 4G$\mathrm{J})$. Normally, however, the TV denoising method is well able to differentiate horizontal cells or vessels and even to recognize target cells, which are positioned immediately above a horizontal cell or a vessel. This is exemplarily shown in Figure 4G-J as well.
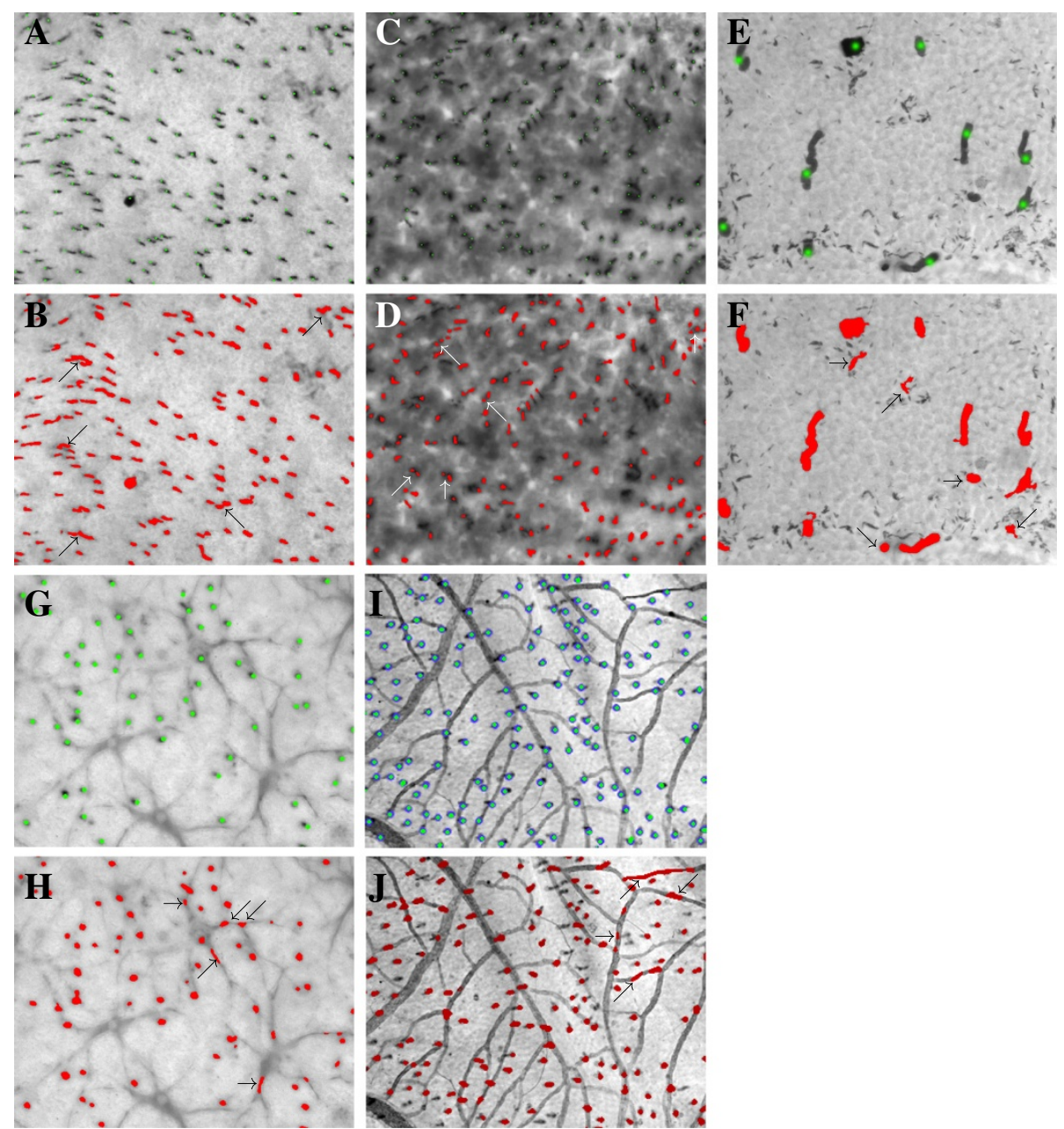

Figure 4 Typical errors in automatic counting. Sample clips with manually counted photoreceptors (tagged with green or blue dots, respectively) $(A, C, E, G, I)$ will be compared with automatic counts obtained with Algorithm 1 (counted features dyed in red) $(B, D, F, H, J)$. In the latter, the arrows point to occasional artifactual errors. (A,B) Lynx, No. 8: Adjacent cells have been merged and counted as a single one. (C,D) Pangolin, No. 12: Single cells are counted twice. (E,F) Agouti, No. 16: Background features (clustered melanin granules) and outer segment fragments are counted as cells. (G,H) Jaguar, No. 11: Parts of horizontal cells in the background are counted as S-cones. Note that, however, S-cones and horizontal cells are generally well differentiated, and even S-cones positioned directly above a horizontal cell are correctly recognized (middle left). (I,J) Orangutan, No. 2: Parts of vessels are counted as S-cones. In general, however, S-cones and vessels are well differentiated, and even S-cones positioned directly above a vessel are correctly recognized (left and middle part of the clip). 


\section{Conclusions}

In conclusion, the presented approach provides a reproducible method for the segmentation of labeled cellular objects, in particular retinal photoreceptors from grey scale micrographs. Relatively simple outlines and little overlap between the targets in the projection plane are clearly important preconditions for the current implementation, but within these limitations the procedure is shown to deliver robust results well comparable to those of manual counts by experienced observers. The method is well documented and can be transferred to other tasks including those with more automatization for both image acquisition and further refinements such as from 2D- or 3D-object recognition criteria.

\section{Availability and requirements}

The software, to which applies the GNU General Public License v.2, is stored at the location http:// www.meduniwien.ac.at/counttool/ within the archive cacount tool 2013 02 08.zip. Its execution requires a current version of MATLAB (e.g., v. 7.14.0.739 (R2012a) and higher) together with the MATLAB Image Processing Toolbox (e.g., v. 8.0 (R2012a) and higher). Within this environment, the tool runs platformindependently. The code has been written in MATLAB; its listing will be provided as Additional file 1. To use the tool, deflate the zip-archive, include its location as well as location of the image data into the MATLAB path and type the command main. Further details on the usage can be found in the accompanying online documentation.

\section{Appendix}

\section{Rudin-Osher-Fatemi TV denoising: mathematical background}

The Rudin-Osher-Fatemi TV denoising procedure fits into a framework where greyscale images will be modeled by "continuous" rather than by "discrete" mathematical objects. More precisely, a greyscale image will be identified with a function $x(s): \Omega \rightarrow \mathbb{R}$, which is at least bounded and measurable. The commonly used model for capturing an original scene is the equation

$$
I(s)=\mathcal{S}(x(s))+\mathcal{N}(s)
$$

where $x(s): \Omega \rightarrow \mathbb{R}$ is an "ideal" image of the scene, $\mathcal{S}$ is an operator encoding the known systematical errors of the imaging device and $\mathcal{N}(s)$ is a noise term, cf. [22], pp. $60 \mathrm{ff}$., and [23], pp. $7 \mathrm{ff}$. Due to the presence of $\mathcal{N}(s)$, the error in the formal solution of (7),

$$
x(s)=\mathcal{S}^{-1}(I(s))-\mathcal{S}^{-1}(\mathcal{N}(s)),
$$

cannot be controlled by the possible deviations within the captured data $I(s)$ alone. In mathematical terms, the reconstruction of the "denoised" or "smoothed" image $x(s)$ via (8) thus represents an "ill-posed problem", which needs for regularization. In large parts of the literature, consequently, image denoising is performed by minimizing functionals of the type

$$
F(x)=\int_{\Omega}(I(s)-\mathcal{S}(x(s)))^{2} d s+\frac{\alpha}{2} \cdot R(\nabla x)
$$

over suitable function spaces, e. g. spaces of Sobolev functions or functions of bounded variation. The first member within $F$, the data fidelity term, aims for a least-square approximation of the captured data $I(s)$ while the second one, the so-called regularization term, has been purposely introduced in order to ensure existence as well as uniqueness of a minimizing solution $x(s)$. The influence of the second term is weighted by a number $\alpha>0$, which is called the regularization parameter. Note that the regularization term depends on the gradient $\nabla x(s)$, thus favorizing a certain edge structure within the minimizing solution $x(s)$. A rigorous mathematical development of this idea relies on a closer analysis of the Euler-Lagrange equation, which must be satisfied as a second-order PDE by the minimizers of (9) as a necessary condition, cf. [22], pp. 64-66.

In the Rudin-Osher-Fatemi TV denoising problem, the operator $\mathcal{S}$ within the data fidelity term is the identity $\mathcal{S}(x(s))=x(s)$. The regularization term, which favors a minimizing solution $x(s)$ with a fairly accentuated edge structure, is taken as $R(\nabla x)=|x|_{T V}$ with

$$
\begin{array}{r}
|x|_{T V}=\sup \left\{\int_{\Omega} x(s)\left(\frac{\partial \psi_{1}}{\partial s_{1}}(s)+\frac{\partial \psi_{2}}{\partial s_{2}}(s)\right)\right. \\
\times d s \mid \psi_{1}(s), \psi_{2}(s): \Omega \rightarrow \mathbb{R} \text { are }
\end{array}
$$

continuously differentiable test functions,

taking zero boundary values and

satisfying $\psi_{1}(s)^{2}+\psi_{2}(s)^{2} \leq 1$ everywhere on $\left.\Omega\right\}$,

and the minimization is performed over all functions of bounded variation on $\Omega$ (for more details, cf. [24], pp. 355 ff., and [25], pp. 175 ff.). Loosely speaking, $|x|_{T V}$ is a measure for the oscillation of the function $x(s)$ and depends on the (generalized) derivatives of $x(s)$ through a duality expression. A closer mathematical analysis reveals that the solution $x(s)$ of the Rudin-Osher-Fatemi TV denoising problem largely preserves the edge structure of the input data $I(s)$. After a suitable discretization, the particular structure of $|x|_{T V}$ allows for the application of highly efficient primal-dual numerical solvers like the one utilized in our present study, cf. again [25], pp. $179 \mathrm{ff}$. 


\section{Additional file}

Additional file 1: Appendix: Documentation of the counting tool.

\section{Competing interests}

The authors declare that they have no competing interests.

\section{Authors' contributions}

PA and CS provided the retinal samples and collected the image data. CS performed the manual counts. KB suggested the application of the TV procedure and programmed the tool. MW performed the numerical experiments and drafted the manuscript. All authors read and approved the final manuscript.

\section{Acknowledgements}

PA and CS have been supported by the Austrian Science Foundation grant I-433 B13 within the E-Rare joint project Rhorcod. KB and MW have been supported by the Austrian Science Foundation within the Special Research Program F 32 "Mathematical Optimization and Applications in Biomedical Sciences" (Graz).

\section{Author details}

${ }^{1}$ Institute for Mathematics and Scientific Computing, University of Graz, Heinrichstraße 36, A-8010 Graz, Austria. ${ }^{2}$ Department of Mathematics, University of Leipzig, P. O. B. 1009 20, D-04009 Leipzig, Germany. ${ }^{3}$ Center for Physiology and Pharmacology, Medical University Vienna,

Schwarzspanierstraße 17, A-1090 Wien, Austria.

Received: 17 December 2012 Accepted: 23 August 2013

Published: 20 October 2013

\section{References}

1. Ahnelt PK, Kolb H: The mammalian photoreceptor mosaic-adaptive design. Prog Retinal Eye Res 2000, 19:711-777.

2. Peichl L: Diversity of mammalian photoreceptor properties: adaptations to habitat and lifestyle? Anat Rec A Discov Mol Cell Evol Biol 2005, 287:1001-1012.

3. Curcio CA, Sloan KR: Packing geometry of human cone photoreceptors: variation with eccentricity and evidence for local anisotropy. Visual Neurosci 1992, 9:169-180

4. Fernández E, Cuenca N, De Juan, J: A compiled BASIC program for analysis of spatial point patterns: application to retinal studies. J Neurosci Methods 1993, 50:1-15.

5. Galli-Resta L, Novelli E, Kryger Z, Jacobs GH, Reese BE: Modelling the mosaic organization of rod and cone photoreceptors with a minimal-spacing rule. Eur J Neurosci 1999, 11:1461-1469.

6. Martinez Mozos, O, Bolea JA, Ferrandez JM, Ahnelt PK, Fernandez E: V-Proportion: a method based on the Voronoi diagram to study spatial relations in neuronal mosaics of the retina. Neurocomputing 2010, 74:418-427.

7. Wässle $\mathrm{H}$, Riemann $\mathrm{HJ}$ : The mosaic of nerve cells in the mammalian retina. Proc Roy Soc B - Biol Sci 1978, 200(1141):441-461.

8. Clérin E, Wicker N, Mohand-Saïd S, Poch O, Sahel JA, Léveillard T: e-conome: an automated tissue counting platform of cone photoreceptors for rodent models of retinitis pigmentosa. $B M C$ Ophthalmol 2011, 11:38. 10.1186/1471-2415-11-38.

9. Filippopoulos T, Danias J, Chen B, Podos SM, Mittag TW: Topographic and morphologic analyses of retinal ganglion cell loss in old DBA/2NNia mice. Invest Ophthalmol Visual Sci 2006, 47:1968-1974.

10. Salinas-Navarro M, Mayor-Torroglosa S, Jiménez-López M, Avilés-Trigueros M, Holmes TM, Lund RD, Villegas-Pérez MP, Vidal-Sanz M: A computerized analysis of the entire retinal ganglion cell population and its spatial distribution in adult rats. Vis Res 2009, 49:115-126.

11. Rudin LI, Osher S, Fatemi E: Nonlinear total variation based noise removal algorithms. Physica D 1992, 60:259-268.

12. Ahnelt PK, Fernández E, Martinez O, Bolea JA, Kübber-Heiss A: Irregular S-cone mosaics in felid retinas. Spatial interaction with axonless horizontal cells, revealed by cross correlation. $J$ Opt SOC Am A: Optics Image Sci Vis 2000, 17:580-588.

13. Ahnelt PK, Schubert C, Kübber-Heiss A, Schiviz A, Anger E: Independent variation of retinal $\mathrm{S}$ and $\mathrm{M}$ cone photoreceptor topographies: a survey of four families of mammals. Vis Neurosci 2006, 23:429-435.

14. Chiu Ml, Nathans J: A sequence upstream of the mouse blue visual pigment gene directs blue cone-specific transgene expression in mouse retinas. Vis Neurosci 1994, 11:773-780.

15. Chambolle A, Pock T: A first-order primal-dual algorithm for convex problems with applications to imaging. J Math Imaging Vis 2011, 40:120-145.

16. Bredies $\mathrm{K}$, Kunisch $\mathrm{K}$, Pock T: Total generalized variation. SIAM J Imaging Sci 2010, 3:492-526.

17. Franek $L$, Franek M, Maurer $H$, Wagner M: A discretization method for the numerical solution of Dieudonné-Rashevsky type problems with application to edge detection within noisy image data. Opt Control App/ Meth 2012, 33:276-301.

18. Laird C, Wächter A: Introduction to IPOPT: a tutorial for downloading, installing, and using IPOPT. Revision No. 1863. Electronically published: http://www.coin-or.org/lpopt/documentation/ (accessed at 11.02.2013).

19. Wächter A, Biegler LT: On the implementation of an interior-point filter line-search algorithm for large-scale nonlinear programming. Math Program Ser A 2006, 106:25-57.

20. Carpenter AE, Jones TR, Lamprecht MR, Clarke C, Kang I, Friman O, Guertin DA, Chang J, Lindquist RA, Moffat J, Golland P, Sabatini DM: CellProfiler: image analysis software for identifying and quantifying cell phenotypes. Genome Biol 2006, 7:R100. 10.1186/gb-2006-7-10-r100.s

21. Danias J, Shen F, Goldblum D, Chen B, Ramos-Esteban J, Podos SM, Mittag $\mathrm{T}$ : Cytoarchitecture of the retinal ganglion cells in the rat. Invest Ophthalmol Vis Sci 2002, 43:587-594.

22. Aubert G, Kornprobst P: Mathematical Problems in Image Processing: Partial Differential Equations and the Calculus of Variations, 2nd ed. New York: Springer; 2006.

23. Chambolle A: Mathematical Problems In Image Processing. Inverse Problems In Image Processing And Image Segmentation: Some Mathematical And Numerical Aspects. ICTP Lecture Notes, II. Trieste: Abdus Salam International Centre for Theoretical Physics; 2000. (electronic).

24. Bredies K, Lorenz D: Mathematische Bildverarbeitung. Einführung in Grundlagen und moderne Theorie. Wiesbaden: Vieweg + Teubner Verlag / Springer Fachmedien Wiesbaden $\mathrm{GmbH} ; 2011$.

25. Chan TF, Shen J: Image Processing and Analysis. Variational, PDE, Wavelet, and Stochastic Methods. Philadelphia: SIAM; 2005.

\section{doi:10.1186/1471-2415-13-59}

Cite this article as: Bredies et al: Computer-assisted counting of retinal cells by automatic segmentation after TV denoising. BMC Ophthalmology 2013 13:59.

\section{Submit your next manuscript to BioMed Central and take full advantage of:}

- Convenient online submission

- Thorough peer review

- No space constraints or color figure charges

- Immediate publication on acceptance

- Inclusion in PubMed, CAS, Scopus and Google Scholar

- Research which is freely available for redistribution 